\title{
A Study on Effective Methods of Visual Inspection for Reused-Parts by Inspectors
}

\author{
Toshiyuki Matsumoto ${ }^{1}$, Hideki Shirai ${ }^{1}$, and Keisuke Shida ${ }^{2}$ \\ ${ }^{1}$ Aoyama Gakuin University 5-10-1 Fuchinobe, Sagamihara-shi, \\ Kanagawa, 229-8558, Japan \\ matsumoto@ise.aoyama.ac.jp \\ ${ }^{2}$ Nagaoka University of Technology 1603-1 Kamitomioka, Nagaoka-shi, \\ Niigata 940-2188, Japan \\ shida@kjs.nagaokaut.ac.jp
}

\begin{abstract}
In order to promote recycling in the recycling industry, it is essential that suppliers perform a strict inspection before shipping the products using reused or recycled parts. The industry is facing pressures to further improve and standardize inspection quality and efficiency on recycled parts or products. This study thus developed an effective visual inspection method for products using reused parts by inspectors at Recycling Plant N, for assuring product quality. First, a survey was conducted to investigate the current situation at the subject plant. Secondly, another set of surveys was conducted for the 13 inspectors. The survey included a video analysis, a questionnaire survey analysis, and an analysis of eye movements using an eye-tracker, and after the analyses, the differences were specified. Thirdly, by considering the inspection method and inspection orders used by the experienced inspectors, appropriate inspection items, inspection order, and inspection method could be devised.
\end{abstract}

\section{Introduction}

Businesses today are facing two significant issues. One is the globalization of the economy, and the other is the growth of ever-increasing global environmental problems. Responding to such trends, businesses are forced to alter their management policies to cope with environmental issues as well as the strengthening of economic growth.

To build a sustainable recycle-oriented society, businesses are working achieve a broad adoption of the 3Rs: Reduce, Reuse, Recycle. In order to promote recycling in the recycling industry, it is essential that suppliers perform a strict inspection before shipping the products using reused or recycled parts. It is also important for them to assure customers of the quality. The industry is thus facing pressures to further improve and standardize inspection quality and efficiency on recycled parts or products.

Two types of inspection systems are currently being implemented: visual inspection which is conducted by quality inspectors, and automatic inspection, which is conducted using instruments. This study deals with the former, that is to say, visual inspection. With conventional visual inspection, quality, efficiency and inspection 
method vary with each inspector. As a result, inspectors may fail to reject defective products. Furthermore, inspection time may vary with each individual. In order to resolve such issues, methods for analyzing visual inspection from the structural viewpoint of the human visual system taking a cognitive scientific approach has been proposed $[1,2,3,4,7]$, and ways to determine efficient visual inspection methods utilizing an industrial engineering approach have also been presented [5,6] However, for structurally-complicated reused parts, it has been often difficult to apply the proposed visual inspection methods.

This study thus aims to develop visual inspection methods for structurallycomplicated reused parts at Recycling Plant $\mathrm{N}$ of an actual supplier. The objectives of this study are to determine appropriate visual inspection orders for covering the necessary inspection items, to create recommendable inspection methods, and to validate the effectiveness of the developed orders and methods upon implementing them at actual inspections.

\section{Subject of Research}

\subsection{Target Parts and Visual Inspection}

This study deals with flash lamps shown in Figure 1, disassembled at the stage of the disassembling process, and the visual inspections for testing the quality of the parts in question.

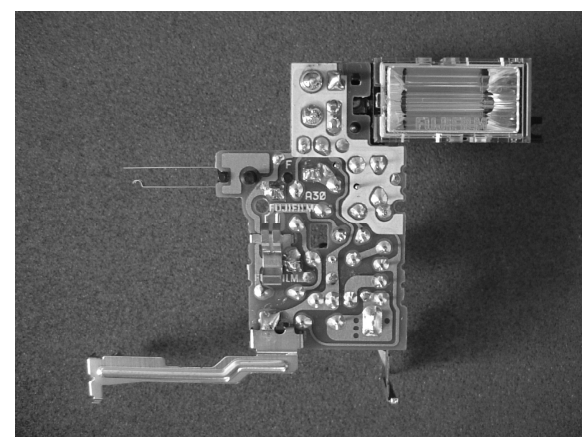

Fig. 1. Target parts

At Plant N, two types of inspections are being conducted to test the quality of flash lamps: performance inspection and appearance inspection. "Performance inspection" tests the flash function of lamps as well as the power distribution of the connecting sections of battery units by sending an electric current. Furthermore, it checks whether products function correctly upon use. Another type of inspection is "appearance inspection", and this checks if there is any change in the products' appearance, for example, if the product is rusted or deformed. Conventionally, both of these inspections were conducted by inspectors. However, as the automation of the inspection process became more popular, performance inspections came to be conducted by 
automatic testers, and also the part of the appearance inspection has come to be conducted automatically by the automated testing system. Currently, visual inspections handle items to be inspected, or inspection items, for subtle deformations or discolorations in appearance, which are hard to detect when using the automatic testing apparatus, and therefore, such visual inspections are conducted by humans.

Table 1. Current inspection and defective items

\begin{tabular}{|c|c|c|}
\hline Item & Defect & Part picture \\
\hline \multirow{3}{*}{ Protector } & Flaw & \\
\hline & Stain & \\
\hline & E rased logo & \\
\hline \multirow{2}{*}{ Reflector } & Flaw & \\
\hline & Stain & \\
\hline \multirow{3}{*}{$\begin{array}{l}\text { Synchro } \\
\text { arm ature }\end{array}$} & D eform ation & \\
\hline & C hange of color & \\
\hline & Rust & \\
\hline \multirow{4}{*}{$\begin{array}{c}\text { B attery } \\
\text { arm ature (-) }\end{array}$} & D eform ation & \\
\hline & F law & \\
\hline & C hange of color & \\
\hline & Rust & \\
\hline \multirow{3}{*}{$\begin{array}{c}\text { B attery } \\
\text { arm ature }(+)\end{array}$} & D eform ation & \\
\hline & C hange of color & \\
\hline & Rust & \\
\hline \multirow{3}{*}{ SM arm ature } & D eform ation & \\
\hline & C hange of color & \\
\hline & Rust & \\
\hline$T$ rans ister & D eform ation & \\
\hline LED & & \\
\hline \multirow[b]{2}{*}{$\mathrm{Xe}$ arm ature } & Change of color & \\
\hline & Rust & \\
\hline \multirow[b]{2}{*}{ Condenser } & D eform ation & \\
\hline & & \\
\hline \multirow[b]{2}{*}{ Trans } & A ttachet sand & \\
\hline & & \\
\hline \multirow{3}{*}{$\mathrm{Xe}$ te $\mathrm{rm}$ inal } & $C \operatorname{rim} p$ & \\
\hline & D eform ation & \\
\hline & & \\
\hline \multirow{3}{*}{$B$ oard } & F law & FनI: \\
\hline & Lack of shape & \\
\hline & & \\
\hline
\end{tabular}

Inspection items and defective items that are currently being examined in the inspections are shown in Table 1. Inspection items range over 10 different items, such as protectors, reflectors, synchronizer sections or connecting sections of battery units. 
Defective items require 25 different types of defects to be detected, such as rust, deformations, scratches or stains.

\subsection{Current Visual Inspection and Its Problems}

Visual inspections of the targeted flash lamps are conducted using a tray which can hold up to 48 flash lamps, as shown in Figure 2. Inspectors hold the trays with both hands and move them, changing the angles at which they are held to check the position of each flash lamp, as well as to inspect the inspection items. At the same time, inspectors move their eyes in certain directions and check if the parts have any defective items.

First, to investigate the current visual inspections, visual inspection processes were video-recorded for 13 inspectors (whom were comprised of 12 experienced inspectors and 1 novice inspector). While the experienced inspectors spent 117 seconds on average to finish inspection of one tray, the novice inspector spent 149 seconds on average; hence it was found that experienced inspectors were more efficient in terms of inspection time.

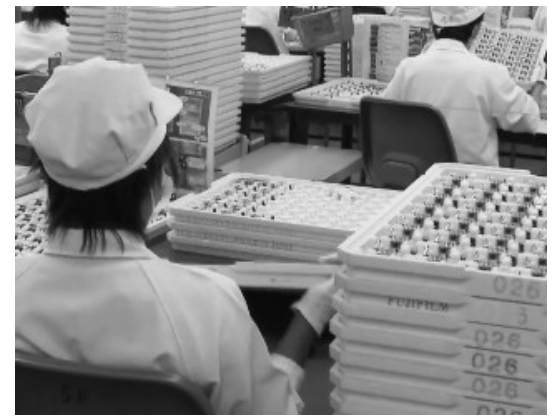

Fig. 2. Picture of inspection process

Second, since each inspector used different methods and had different ideas on defective items, ideal visual inspection methods needed to be established. Basically, inspectors check parts arranged on a tray, looking at the position of each inspection item in order. However, it was found that all inspectors did not take the same approach. Therefore it was found necessary to establish ideal inspection methods by comparing and analyzing characteristics of visual inspections of experienced inspectors who identify defective items at a high rate, as well as the methods of novice inspectors.

\section{Analysis of the Questionnaire Survey on Visual Inspection Items}

To investigate how inspectors conduct visual inspections, first, a questionnaire survey was conducted targeting the 13 inspectors. The questionnaire covered questions in 2 categories: questions regarding inspection orders with the inspection items and questions regarding inspection methods.

After analyzing the result from the questionnaires, as for inspection orders, it was confirmed that the inspection orders were inconsistent, and that there was no 
standardized order. Regarding inspection orders considering positional relationship of parts, it was commonly accepted to go over the inspection items from the same direction consecutively, since that can reduce the number of times the parts are handled, as well as improving and increasing productivity of operations. Also, by checking the important items first and by reexamining the items as time allows, it was hypothesized that inspection quality could be improved.

As for inspection methods, basically the inspectors' eyes moved in a vertical Sshaped path, although according to some inspection items, some moved their eyes in vertical or horizontal directions. Thus it was found that inspection angles or directions were not consistent either. For this, the authors considered it was necessary to obtain knowledge and views from inspectors who are experienced and can identify defective items at a high rate, analyzing the result of the eye-tracking test which will be discussed later.

\section{Analysis of Visual Inspection Items through Analyzing Inspectors' Eye Movements}

After analyzing the video-recorded operations and questionnaire, it was found that experienced inspectors and novice inspectors detected defective items in a different manner. To verify this finding, Eye Mark Recorder, or an eye-tracking apparatus (called "Eye Camera", produced by NAC Image Technology Inc.) was used, and the operators' eye movement was analyzed.

To conduct this test, 3 examinees were selected from 3 different skill levels of inspectors: one from the most experienced group who boasted the highest defectdetecting skills (a.k.a. the advanced level inspector), one from the semi-experienced group (a.k.a. the intermediary level inspector), one untrained inspector (a.k.a. the novice inspector).

In the experiment, examinees were asked to conduct inspections for 5 trays wearing the Eye Cameras. Operators' eye movement was tracked during the tests. The movement was then analyzed for defect-free trays and trays with defective parts.

The trajectories shown in Figure 3 show the tracked eye movements from each of the 3 examinees. They also show the fixation points during the inspections of the defect-free trays. For the advanced level inspector, fixation points moved at an almost certain angle, as is shown in the trajectory. Studying the field of view monitor, the inspector did not move the head, and instead, handled the tray skillfully so as to minimize the inspection scanpaths. Movement speed was almost constant and not as variable as the others. The inspector's eyes moved at a certain, constant speed.

On the other hand, the intermediary level and novice inspectors moved their eyes at winding angles, and checked the inspection items, as shown in their inspection scanpaths. Looking at the trajectory from the novice inspector in particular, the inspection scanpaths were not followed and adjusted according to the focal point distance, which resulted in overlooking certain defects, and thus this inspector's visual inspection method was not consistent, as can be analyzed from the results. This seems to be because the inspector was poorly-trained. The field of view monitor showed that this 

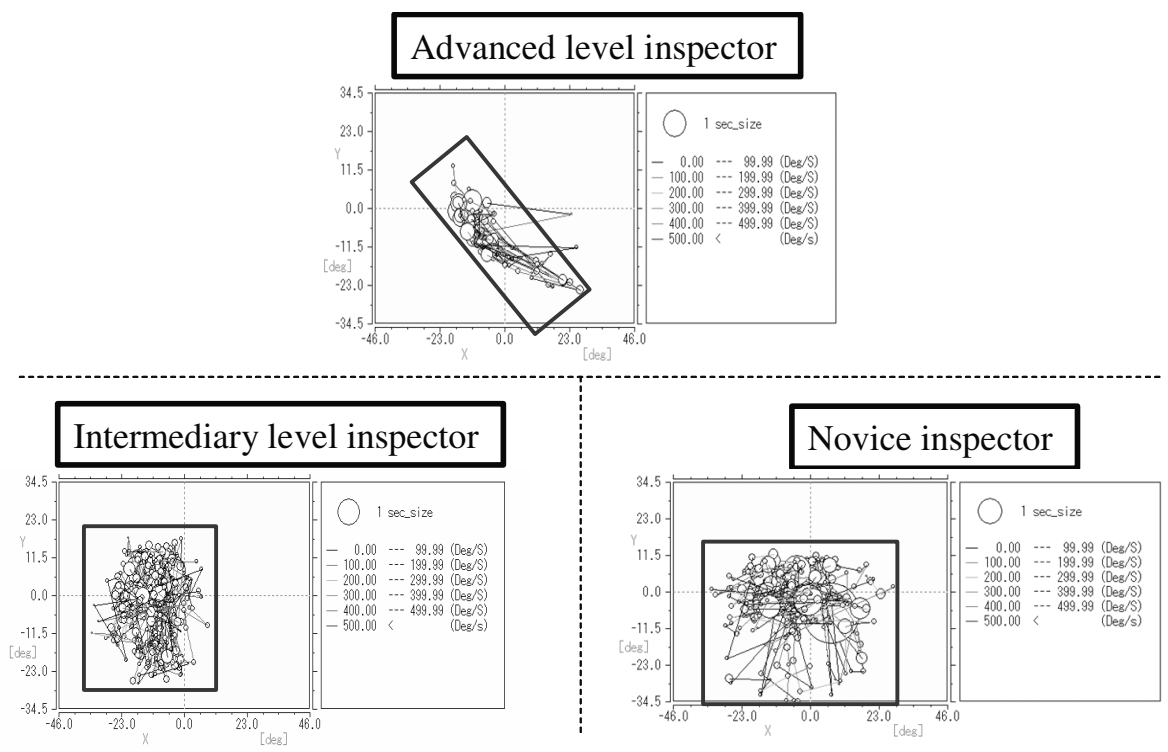

Fig. 3. The tracked eye movements from each of the examinees

inspector did not move the head or the trays and only moved the eyes, so the eyes' moving range was rather wide in this case.

Studying the visual inspection method of the advanced level inspector, it became clear that the ocular movement or the eye movement followed the targets with saccades. It was also found that the targets that the inspector's eyes followed were not single inspection items but rather several inspection items located at the lighted portion of the tray, using the peripheral visual field. In other words, the advanced level inspector used peripheral view to detect anything improper with the parts, and with saccadic eye movements, identified defects with the parts. Furthermore, when the defects were identified, the inspector's fixation points were not fixed on the defective positions themselves, although the defects were within inspector's peripheral visual field.

As for the intermediary and novice inspectors, their eyes moved to scan for detects on the targets upon inspection. When they moved from one target to another, one saccade was not enough for processing the necessary visual information to detect defects, thus their eye movement involved a series of quick saccades. However, as the inspectors continued inspection, their eye movement involved less and less saccades because of eyestrain, and their movement speed became unstable. Eyes that are fixed for a shorter period for each target resulted in overlooking defects or erroneous inspection.

\section{Determining Inspection Items, Order and Implementation}

Analyzing the results of the experiment above, proper visual inspection order should consider the elements for successful visual inspections identified in the experiment. 
Upon determining inspection order, inspection angles at which inspection is to be conducted most efficiently for each inspection item must be clarified. At the same time, the number of handling steps must be considered to create efficient inspection flow. To consider these, visual inspection items and defective items to be checked during an inspection must be listed, and then the appropriate inspection order can determined.

First, visual inspection items and defective items to be dealt in each process of the inspection must be clarified. Possible defective items to be detected for the flash lamps were listed studying the product's specifications, interviewing inspectors, and considering properties of materials used for the each inspection position of the part. The items were cross-checked with the items to be covered in the specification, and an exhaustive list which includes all the possible defective items was then formed. As for the materials of the parts, plastic and other 3 types of metal (copper, aluminum, and lead) were used. Possible defects were considered for each type of material, such as defects uniquely observed for reused parts, deterioration-related defects such as rust or stains, and deformation defects such as dents or flexions.

From the created exhaustive list which covers the defective items, visual inspection items and defective items to be inspected by automatic testers were eliminated, and the existing visual inspection and defective items were streamlined. Automatic testers mainly deal with deformation defects, although visual inspection items undetectable

Table 2. Finalized inspection and defective items

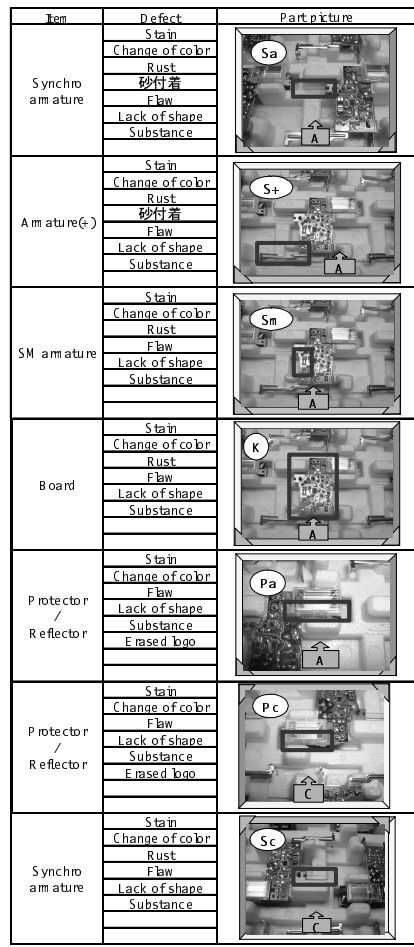


by a camera can only be inspected by the human eye. Such special cases were also taken into consideration and after reviewing the list, a new list covering visual inspection and defective items to be inspected was created.

Next, inspection order for visual inspection items to be covered for each scan is to be determined. There are 7 angles to be scanned in an inspection, and by conducting inspection from the same scan angle successively, the number of handling steps can be reduced. Possible inspection orders were thus considered and then listed in a matrix. Upon creating the matrix, 3 types of constraints were imposed. (1) Inspection items which can be inspected from the same angles should be covered successively. The angle at which the tray is originally placed is the first inspection angle. (2) Protectors and reflectors require 2 scans for their inspection items; inspection items serve as switching points of inspection angles. (3) Important inspection items that are most likely to have defects must be checked first. Importance was determined based on the previous data, calculated from the number of detected defective parts in all of the parts put into the visual inspection processes. Using the matrix, inspection order was finalized, and the finalized inspection order is shown on Table 2.

The inspection order and the visual inspection method discussed in the previous chapter were adopted at the targeted inspection process. Prior to the implementation of the new inspection method and order, all the inspectors were given a training session using a new manual which explains the new inspection items. Inspections were then conducted following the new inspection order. Now, we are examining the undetected defect probabilities in the visual inspection processes.

\section{Conclusions}

This study developed an effective visual inspection method for products using reused parts at Recycling Plant N, for assuring product quality. First, a survey was conducted to investigate the current situation at the subject plant. After the survey, problems were identified, and in order to solve the problems, another set of surveys was conducted for the 13 inspectors (12 experienced inspectors with a high defect-detecting rate and one novice inspector). The survey included a video analysis, a questionnaire survey analysis, and an analysis of eye movements using an eye-tracker, and after the analyses, the differences were specified. By considering the inspection method and inspection orders used by the experienced inspectors, appropriate inspection items, inspection order, and inspection method could be devised. The devised inspection method was applied for the target processes.

The authors would like to examine the result of the devised inspection method and further develop effective training methods for activating the peripheral visual field as experienced inspectors do upon inspection, as an issue in the future.

\section{References}

1. Akutsu, M.: Ergonomic Design Approach for Improving Industrial Visual Inspection Study on Structure and Optimum Feeding Rate in Visual Sorting Inspection. The Japanese Journal of Ergonomics 31, 426-427 (1995) 
2. Chan, A.H.S., Courtney, A.J.: Effects of object size and inter-object spacing on peripheral object detection. International Journal of Industrial Ergonomics 25(4), 359-366 (2000)

3. Kubota, S., Ohkura, M.: IIDA Hiroyasu: Eye Movement in Visual Inspection. The Journal of science of labor 57(12), 623-631 (1981)

4. Moeck, M.: Lighting design based on luminance contrast. International Journal of Lighting Research and Technology 32(2), 55-63 (2000)

5. Sasaki, A.: A method of Visual Inspection with Peripheral Vision [2]. IE Review 46(5), 61$68(2005)$

6. Sasaki, A.: A method of Visual Inspection with Peripheral Vision [1] - Introduction of Outline. IE Review 46(4), 65-70 (2005)

7. Takeoka, K., Tagawa, S., Akutsu, M.: Study on Optimum Speed of Work in Visual Inspection Conducted by Tact Inspection System -Study on Structure and Optimum Speed of Work (Part III). Journal of Japan Industrial Management Association 42, 1-7 (1991) 